\section{PRADS 2010}

$11^{\text {th }}$ International Symposium on Practical Design of Ships and Other Floating Structures

Rio de Janeiro, 19-24 September 2010

www.prads2010.org.br

\section{FPSO Research Forum 2010}

\section{$25^{\text {th }}$ FPSO JIP Week}

Aberdeen, Scotland, 11-15 October 2010

www.fpsoforum.com/upcoming.html

\section{LNG Tech Global Summit 2010}

Rotterdam, Netherlands, 19-20 October 2010

www.lngsummit.com/

\section{SOBENA 2010}

XXIII Brazilian Conference on Maritime Transport, Shipbuilding and Offshore Engineering

Rio de Janeiro, Brazil, 25-29 October 2010

www.sobena.org.br

\section{SNAME 2010 Annual Meeting \& Expo}

Seattle/Bellevue, Washington, USA, 03-05 November 2010 www.sname.org

\section{DOT 2011}

Deep Offshore Technology

New Orleans, USA, 1-3 February 2011

www.bvents.com/event/251991-dot-deep-offshoretechnology

\section{DINAME 2011}

$14^{\text {th }}$ International Symposium on Dynamic Problems in Mechanics

São Sebartião, São Paulo, Brazil, 13-18 March 2011

www.abcm.org.br/diname2011

\section{IWWWFP 2011}

$25^{\text {th }}$ International Workshop on Water Waves and Floating Bodies

Athens, Greece, 17-20 April 2011

www.iwwwfb.org/Workshops/26.htm

\section{OTC 2011}

$41^{\text {st }}$ Offshore Technology Conference

Houston, USA, 2-5 May 2011

www.otcnet.org/2011

\section{OMAE 2011}

$30^{\text {th }}$ International Conference on Ocean, Offshore and Arctic Engineering

Rotterdam, Netherlands, 19-24 June 2011

www.asmeconferences.org/omae2011

\section{ISOPE 2011}

$21^{\text {st }}$ International Offshore and Polar Engineering Conference Maui, Hawai, USA, 19-24 June 2011

www.isope.org

\section{ITTC 2011}

$26^{\text {th }}$ International Towing Tank Conference

Rio de Janeiro, Brazil, 28 August-03 September 2011

www.laboceano.coppe.ufrj.br 